\title{
Medicinal plants conservation and cultivation by traditional medicine practitioners (TMPs) in Aiyedaade Local Government Area of Osun State, Nigeria
}

\author{
A.T Oladele ${ }^{1}$ G.O Alade $^{2}$ and O.R Omobuwajo ${ }^{2}$ \\ ${ }^{1}$ Department of Pharmacognosy, Obafemi Awolowo University, Ile-lfe, Nigeria. \\ kunleladele@yahoo.com, aoladele@oauife.edu.ng \\ ${ }^{2}$ Department of Pharmacognosy and Herbal Medicine, Niger Delta University, Wilberforce \\ Island, Nigeria.
}

\begin{abstract}
The contribution of Traditional Medicine Practitioners (TMPs) to conservation of medicinal plants and the acceptability of cultivated plants as alternative to wild sources were studied in Aiyedade Local Government area of Osun State, Nigeria. 55 TMPs were randomly selected for interview from 120 registered TMPs in the study area. $94.5 \%$ of the respondents were aware of plant species which had become scarce. Results revealed that $41.3 \%$ of plants cultivated in their homesteads were herbs, Shrubs (29.3\%), Trees(18.7\%) and climbers(10.7\%). 51.6\% of plants from wild sources were trees while $44.1 \%$ of plants bought from herb markets were trees. Correlation between scarcity of medicinal plants and travel distance to collect plant materials by TMPs was positive $(0.278, \rho<0.05)$, problems associated with cultivation such as land and collection from the wild was equally positive $(0.358, \rho<0.01)$. Sustainable harvesting and deliberate cultivation have been proposed to ensure continued supply of medicinal plants to meet the health care needs of rural dwellers in the study area.
\end{abstract}

Keywords: Traditional Medicine Practioners (TMPs), Medicinal plants, Conservation, Cultivation, Aiyedade LGA (Osun state).

\section{INTRODUCTION}

Medicinal plants play a crucial role in health care needs of people around the world especially in developing countries (Bekalo et al., 2009, Rao et al., 2004). About $80 \%$ of the population of most developing countries still depend on the use of traditional medicine derived from plants (Cunningham, 1993). People living in remote areas depend more extensively on traditional medicine as modern systems are out of reach (Mahonge et al., 2006, de Silva 1997, Bodeker, 1994, Sofowora, 1993, Bhat et al., 1990). Medicinal plants play a key role in the development and advancement of modern studies by serving as a starting point for the development of novelties in drugs (Pramono, 2002). Approximately $25 \%$ of drugs used in modern Pharmacopoiea are derived from plants and many others are synthetic analogues built on prototype compounds isolated from plants. It was estimated that the total number of medicinal plants in international trade is about 2500 species (Schippman et al., 2002). A large proportion of these plants are obtained from wild sources and the forest in particular
(Gupta, 1986). The demands of the majority of the people in developing countries for medicinal plants have been met by indiscriminate harvesting of spontaneous flora including those in forests. Over exploitation of these wild sources has led to many species being extinct, threatened or endangered (Omobuwajo et al., 2008). Indiscriminate exploitation has been the trend for decades in developing countries and therefore generate conservation issues for these important plants. Population explosion in developing countries has exerted undue pressure on frequently used medicinal plants and natural resources in general. As markets increases for medicinal plants products, wild population are been depleted.

Sustainable practice of traditional medicine and supply of plant materials for drug development are hinged heavily on deliberate and concerted efforts to conserve these indigenous plants. Numerous medicines have been derived from the knowledge of tropical forest people and clearly there will be more in the future. This alone is reason enough for any and all programmes to be concerned with the 
conservation, development, and protection of tropical forest regions. Since human needs and problems are a primary component of any conservation program. It is therefore necessary that systematic cultivation of medicinal plants be introduced in order to conserve biodiversity and protect threatened species.

The present study was carried out to document the activities of the Traditional Medicine Practioners (TMP's) in conservation of medicinal plants in Aiyedade local government area of Osun State, Nigeria.

\section{MATERIALS AND METHODS}

Study Area: Aiyedade local government area is located in the western part of Osun State of Nigeria.
It has a population of 150,392 (Federal Government of Nigeria, 2007) and is bounded by Isokan, Irewole and Aiyedire local governments to the east and Ife North local government to the west (Fig.1). The people belong to the Yoruba speaking tribe and are mainly peasant/subsistence farmers predominantly living in rural communities, which informed their dependence on plants for their healthcare needs. For the purpose of the survey, five communities (Gbongan, Odeomu, Wakajaiye, Orile Owu and Ogbaaga) were randomly selected for questionaire administration in the study area. Traditional Medicine Practitioners (TMPs) were visited in their premises for inventory and identification of plants cultivated in their home gardens.

Fig. 1: Map of Aiyedaade Lga showing the study areas

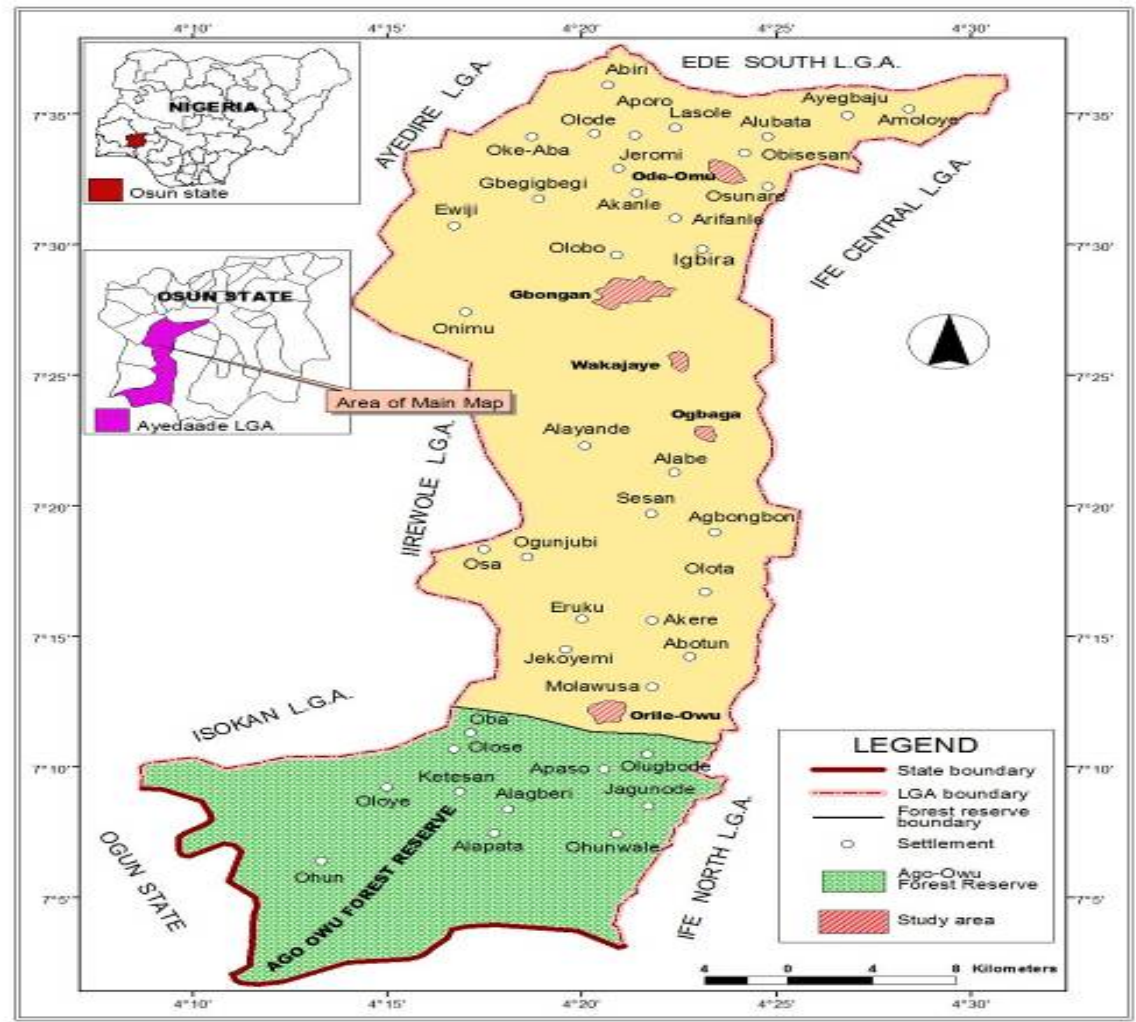

Registered TMPs with the Osun State Ministry of Health, Osogbo, were randomly selected from Ayedaade Local Government Area. 55 of the 120 registered TMPs were selected, visited and interviewed representing $45.8 \%$ of the sample population. The process of data collection include identification and specimen collection of the medicinal plants cultivated in the premises of the TMPs. Information concerning the distribution, propagation method, conservation strategies, uses 
and local names of the plant were obtained where possible from TMPs. The resulting data was analyzed using simple percentages (frequencies) and bivariate Pearson correlation analysis using SPSS 16.0, (2007). The plants were scientifically identified using reference books and comparison of plant specimen collected with voucher specimens at the herbarium of Botany Department, Obafemi Awolowo University, Ile-Ife, Nigeria. The research instrument was a structured questionaire administered by interpreting into Yoruba language which was most readily understood by the TMPs. Informed consent of the TMPs were obtained to make the information public before they were interviewed. Stipends were offered in appreciation of the time spent in taking us round their homesteads and responding to our questions.

\section{RESULTS}

A significant percentage $(80.7 \%)$ of the TMPs were over the age of 40 (Table 1), this showed that the

Table 1: Age, education and experience of Traditional Medicine Practitioners in Ayedaade LGA, Osun State, Nigeria

\begin{tabular}{|c|c|c|c|c|c|c|c|c|}
\hline $\begin{array}{l}\text { Age of } \\
\text { TMPs } \\
\text { (Years) }\end{array}$ & Frequency & $\begin{array}{c}\text { Percentage } \\
\%\end{array}$ & $\begin{array}{c}\text { Education } \\
\text { of TMPs }\end{array}$ & Frequency & $\begin{array}{c}\text { Percentage } \\
\%\end{array}$ & $\begin{array}{c}\text { Experience } \\
\text { of TMPs }\end{array}$ & Frequency & $\begin{array}{c}\text { Percentage } \\
\%\end{array}$ \\
\hline $21-30$ & 4 & 7.5 & $\begin{array}{l}\text { No Formal } \\
\text { education }\end{array}$ & 25 & 47.0 & $\begin{array}{l}\text { Inheritance } \\
\text { (From birth) }\end{array}$ & 13 & 24.5 \\
\hline $31-40$ & 8 & 14.9 & $\begin{array}{l}\text { Primary } \\
\text { school }\end{array}$ & 10 & 18.9 & $0-5 y e a r s$ & 1 & 1.9 \\
\hline $41-50$ & 9 & 17.0 & $\begin{array}{c}\text { Secondary } \\
\text { school }\end{array}$ & 13 & 24.5 & Above 5yrs & 34 & 64.2 \\
\hline $51-60$ & 7 & 13.2 & $\begin{array}{c}\text { Above } \\
\text { secondary } \\
\text { school }\end{array}$ & 7 & 13.2 & $\begin{array}{c}\text { No } \\
\text { response }\end{array}$ & 8 & 12.7 \\
\hline $\begin{array}{l}\text { Above } \\
60\end{array}$ & 27 & 50.5 & - & - & - & - & - & - \\
\hline Total & 55 & 100 & Total & 55 & 100 & Total & 55 & 100 \\
\hline
\end{tabular}

Table 2 showed that about $26.4 \%$ of the TMPs interviewed, treat between 21 and 40 patients per day while $13.2 \%$ have above 40 patients daily. This demonstrates the fact that majority of the populace in rural areas still patronise TMPs. More than half of the informants have no apprentices as at the time of the interview. The reason given for this lack of apprenticeship was that people now go for formal education and that those that would have combined it have no time. This is corroborated with the age distribution of TMPs in Table 1.

The major means of sourcing medicinal plants materials among TMPs is by self collection from the wild/home gardens $(86.8 \%)$ and purchase from herb younger generation are not many in traditional medicine practice due to rural urban migration. About $47.0 \%$ did not receive any formal education in organised school setting, while $24.5 \%$ of the informants attended high school and $18.9 \%$ had primary school education. Few elites with tertiary education (13.2\%) were TMPs who in most cases are retired civil servants that inherited the skill from their progenitors. Most of the TMPs claimed they inherited the healing skills from their parents or relatives who are knowledgeable and others through apprentism. In some cases the knowledge is restricted within the family members and forbiden to share the knowledge with non-family members. About $88.7 \%$ of the informants have above 5 years of training/experience which is equivalent to the minimum for training a medical doctor. Improved skill has been associated with number of years of practice. 
Agric. Biol. J. N. Am., 2011, 2(3): 476-487

Table 2: Daily patients call and Apprentice number per TMP in Ayedaade LGA, Osun State, Nigeria

\begin{tabular}{|c|c|c|c|c|c|}
\hline Daily patients call per TMPs & Frequency & Percentage \% & Number of apprentice per TMPs & Frequency & Percentage \% \\
\hline Below 20 patients & 29 & 54.7 & Nil & 31 & 07 \\
\hline $21-40$ & 14 & 26.4 & $3-4$ & 05 & 13.2 \\
\hline $41-50$ & 2 & 3.8 & 5 and above & 04 & 7.6 \\
\hline Above 50 & 5 & 9.4 & No response & 08 & 14.9 \\
\hline No response & 5 & 9.4 & $\mathbf{1 0 0}$ & $\mathbf{5 5}$ & $\mathbf{1 0 0}$ \\
\hline
\end{tabular}

Table 3: Medicinal plants sources, reasons for buying herbs and reasons for medicinal plants cultivation by TMPs in Ayedaade LGA, Osun State, Nigeria

\begin{tabular}{|c|c|c|c|c|c|c|c|c|}
\hline $\begin{array}{l}\text { Sources of med. } \\
\text { plants }\end{array}$ & Freq. & $\%$ & Reasons for buying herbs & Freq. & $\%$ & $\begin{array}{l}\text { Reasons for } \\
\text { cultivation }\end{array}$ & Freq. & $\%$ \\
\hline $\begin{array}{l}\text { Self collection from } \\
\text { wild sources }\end{array}$ & 46 & 86.8 & Scarcity due to overexploitation & 31 & 58.5 & $\begin{array}{l}\text { Easily cultivated } \\
\text { plants }\end{array}$ & 9 & 17.0 \\
\hline $\begin{array}{l}\text { Buy from herb } \\
\text { vendors }\end{array}$ & 6 & 11.3 & It saves time & 11 & 20.8 & $\begin{array}{l}\text { Frequently used } \\
\text { plants }\end{array}$ & 35 & 66.0 \\
\hline No response & 3 & 5.5 & $\begin{array}{l}\text { Plants that do not grow in the } \\
\text { neighbourhood forests }\end{array}$ & 3 & 5.7 & $\begin{array}{l}\text { Very costly/scarce in } \\
\text { the market }\end{array}$ & 1 & 1.9 \\
\hline - & - & - & Other reasons & 2 & 3.8 & $\begin{array}{l}\text { Easily perishable } \\
\text { plants }\end{array}$ & 2 & 3.8 \\
\hline - & - & - & No response & 8 & 14.9 & $\begin{array}{l}\text { Plants that must be } \\
\text { used fresh }\end{array}$ & 2 & 3.8 \\
\hline- & - & - & - & - & - & No response & 6 & 11.3 \\
\hline Total & 55 & 100 & Total & 55 & 100 & Total & 55 & 100 \\
\hline
\end{tabular}

About $83.7 \%$ of the TMPs purchase scarce medicinal plants daily, $65.5 \%$ purchase medicinal plants worth at least 6 U.S dollars (about N1,000 Nigerian naira) daily (Fig.2 and Table 8). 


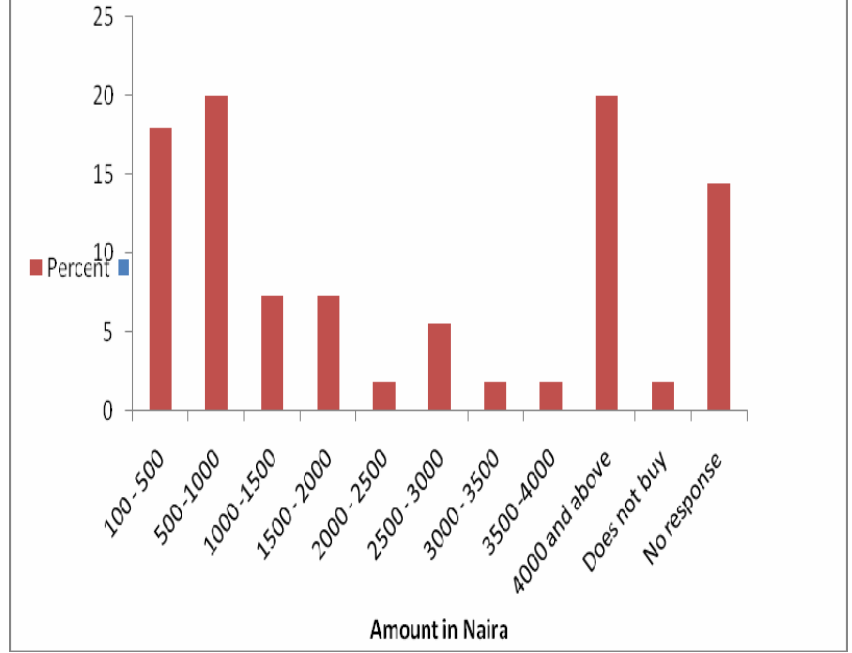

Fig 2: Average daily purchase of herbs by Traditional Medicine Practitioners in Ayedaade LGA, Osun State, Nigeria

Not less than $92.7 \%$ of the TMPs travel considerable distances $(2 \mathrm{~km}$ - above $8 \mathrm{~km})$ before they could obtain some important plant resources needed for herbal preparations due to scarcity. Scarcity of medicinal plants has been linked to uncontrolled deforestation by human activities in tropical forest regions worldwide. $94.5 \%$ of the interviewed TMPs were aware of plants previously available in their adjoining forests which have disappeared into the far forest (Table 4 and Table 7), this implies that some plant species are becoming scarce and some may be on the verge of extinction (Omobuwajo et al. 2008, Kisangau et al 2004, Tomlison 1998, Quedraogo 1995). Also, the high patronage of patients at herbal homes has placed pressure on the plant resources (Table 2.). There is no considerable awareness $(77.4 \%)$ that medicinal plants are being cultivated on a large scale among the TMP's in the study area,

Lack of land was observed by the informants as the most significant constraint to medicinal plant cultivation in the study area, represented by (47.3\%) of TMPs (Fig.4). About $21.8 \%$ of the TMPs in the study population had no time to maintain such farms; probably they have to be present in their premises always.

Bivariate Pearson correlation analysis gave a negative $(-0.284, \rho<0.05)$ correlation between medicinal plants cultivation and TMPs willingness to buy from cultivated sources, therefore the TMPs that purchase herb materials from herb sellers do less planting in their premises. However, increase in distance to collect herbs from wild sources has encouraged TMPs to buy from herb sellers, hence, higher average daily budget on herbs by TMPs as reflected in the positive correlation $(0.382, \rho<0.01)$. Similarly, scarcity of medicinal plants in the wild also resulted in increased average daily budget of TMPs on herbs $(0.355, \rho<0.01)$, and increased distance travelled in the forest for raw materials $(0.278, \rho<$ $0.05)$. Problems associated with medicinal plants cultivation such as land and availability of propagules has made the adjoining forests a good option for raw materials collection $(0.358, \rho<0.01)$ by the TMPs. It was also observed that the educated elites among the TMPs received more patients in their clinics, correlation between education of TMPs and patient patronage was positive $(0.352, \rho<0.01)$.

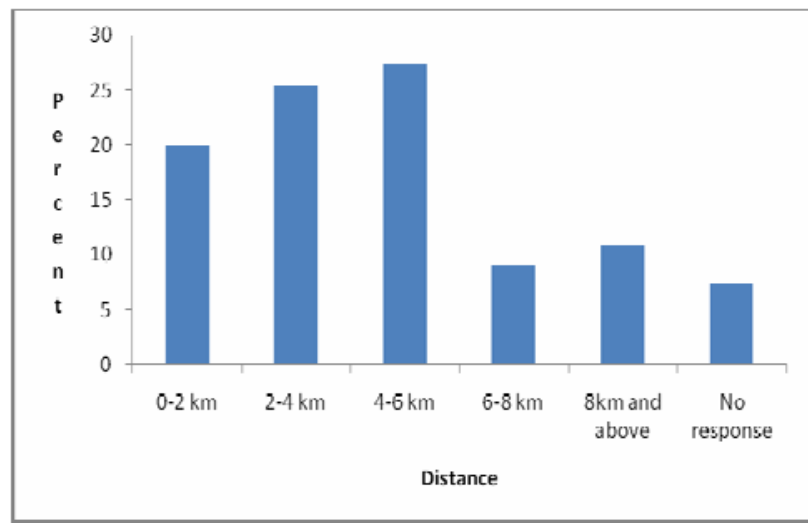

Fig 3 : Distance travelled by Traditional Medicine Practitioners to collect medicinal plants in Ayedaade LGA, Osun State, Nigeria

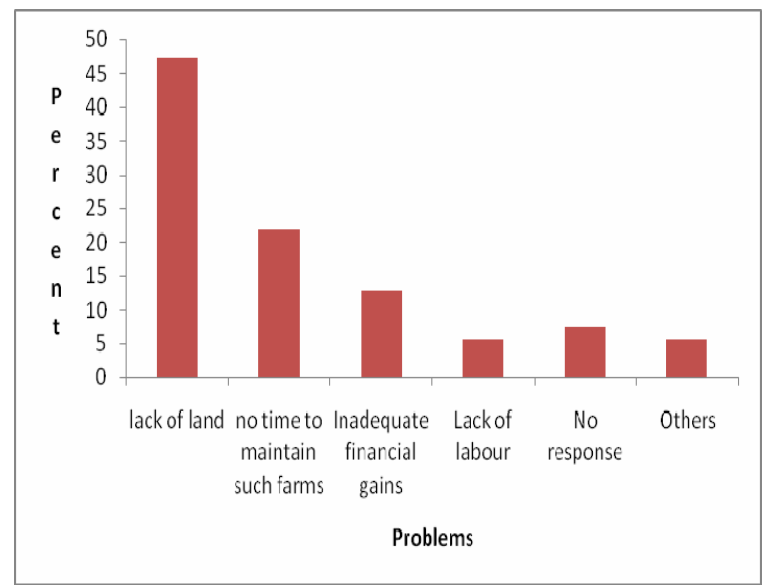

Fig 4: Problems associated with medicinal plants cultivation among the Traditional Medicine Practitioners in Ayedaade LGA, Osun State, Nigeria 
Table 4: Scarcity and existence of medicinal plants farm awareness, and willingness to patronise such farms by TMP's in Ayedaade LGA, Osun State, Nigeria

\begin{tabular}{|c|c|c|c|c|c|c|}
\hline \multirow[t]{2}{*}{ Awareness } & \multicolumn{2}{|c|}{ Scarcity } & \multicolumn{2}{|c|}{ Existence } & \multicolumn{2}{|c|}{ Willingness to buy } \\
\hline & Freq. & $\%$ & Freq. & $\%$ & Freq. & $\%$ \\
\hline Yes & 52 & 94.5 & 6 & 11.3 & 48 & 86.8 \\
\hline No & 1 & 1.9 & 41 & 77.4 & 4 & 7.7 \\
\hline No response & 2 & 3.8 & 8 & 14.9 & 3 & 5.5 \\
\hline Total & 55 & 100 & 55 & 100 & 55 & 100 \\
\hline
\end{tabular}

It is obvious that TMPs attempt to conserve plant resources for their immediate and future need by maintaining medicinal plant gardens around their homes / premises (Table $5 \& 6$ ), this is obviously inadequate compared to their needs. In all the home gardens visited, 75 medicinal plants genera distributed into 42 families were observed to be cultivated by the TMPs in their premises (Table 6). The plant forms (Table 5) showed that $41.3 \%$ of plants cultivated by TMPs in their premises in the study area were herbaceous and annual/biannual plants such as Kalanchoe crenata, Bryophyllum pinnatum, Abrus precatorious, Ocimum spp., and Vernonia amygdalina. Perennial shrubs such as Senna alata and Jatroha spp. were $29.3 \%$, Trees used in the treatment of prevalence diseases like malaria such as Azadirachta indica and Croton zambesicus represent $18.7 \%$, while climbers used in fertility cases like Pergularia daemia,Mormodica charantia and Parquetina nigriscens form $10.7 \%$ of plants grown by the TMPs. Medicinal plants from wild sources for traditional medicine showed 64 genera from 40 families (Table 7) distributed into $51.6 \%$ trees, such as Khaya senegalensis, Morinda lucida, Alstonia boonei, Rauwolfia vomitoria and Tetrapleura tetraptera. These trees are commonly used in the treatment of malaria which is a prevalence disease in the study area, apparently these tree species do not regenerate easily as a result of annual bush burning and shifting cultivation system of agriculture practiced in the study area. All these factors have forced the TMPs to travel farther into the adjoining forests to source their plant materials. $20.3 \%$ were shrubs, $17.2 \%$ herbs and $10.9 \%$ climbers, this phenomenon will obviously hasten the extinction of the frequently used species that are not harvested in sustainable manner. Plant materials purchased in the market from the survey were 34 genera from 25 families which are mostly sourced from the wild by the suppliers and represent $44.1 \%$ (Trees), $26.5 \%$ (herbs), $20.6 \%$ (Shrubs) and $8.8 \%$ climbers (Table 8 ).

Table 5: Plant forms of medicinal plants cultivated by TMPs in their homesteads, wild and market sources in Ayedaade LGA, Osun State, Nigeria

\begin{tabular}{|c|c|c|c|c|c|c|}
\hline & \multicolumn{2}{|c|}{$\begin{array}{c}\text { Plants grown in TMPs } \\
\text { premises }\end{array}$} & \multicolumn{2}{c|}{ Plants from wild sources } & \multicolumn{2}{c|}{$\begin{array}{c}\text { Plants purchased from the } \\
\text { markets }\end{array}$} \\
\hline Plant forms & Frequency & \% & Frequency & \% & Frequency & \% \\
\hline Herbs & 31 & 41.3 & 11 & 17.2 & 09 & 26.5 \\
\hline Shrubs & 22 & 29.3 & 13 & 20.3 & 07 & 20.6 \\
\hline Trees & 14 & 18.7 & 33 & 51.6 & 15 & 03 \\
\hline Climbers & 08 & 10.7 & 07 & 10.9 & $\mathbf{3 4}$ & 8.1 \\
\hline Total & $\mathbf{7 5}$ & $\mathbf{1 0 0}$ & $\mathbf{6 4}$ & $\mathbf{1 0 0}$ & $\mathbf{3 4}$ & $\mathbf{1 0 0}$ \\
\hline
\end{tabular}


Table 6: Medicinal plants grown by TMPs in Ayedaade LGA, Osun State, Nigeria.

\begin{tabular}{|c|c|c|c|c|c|}
\hline No & Name & Family & $\begin{array}{l}\text { Voucher } \\
\text { number }\end{array}$ & $\begin{array}{l}\text { Cultivation } \\
\text { frequency }\end{array}$ & $\begin{array}{l}\text { Local name } \\
\text { Yoruba }\end{array}$ \\
\hline 1 & Croton zambesicus Muell. Arg & Euphorbiaceae & UHI (2575) & 24 & Ajeobale \\
\hline 2 & Ocimum gratissimum $\mathrm{L}$ & Lamiaceae & UHI (14849) & 23 & Efinrin foromoba \\
\hline 3 & Vernonia amygdalina Schreber & Asteraceae & UHI (6532) & 21 & Ewuro \\
\hline 4 & Jatropha curcas L. & Euphorbiacae & UHI (8586) & 18 & Lapalapa, \\
\hline 5 & Senna alata $\mathrm{L}$ & Caesalpinaceae & UHI (13918) & 18 & Asunran pupa \\
\hline 6 & Jatropha gossypifolia L & Euphorbiaceae & UHI (2700) & 17 & Lapalapa pupa \\
\hline 7 & Kalanchoe crenata (Andr.) Haw & Crassulaceae & $\mathrm{UHI}(407)$ & 16 & Odundun \\
\hline 8 & Abrus precatorious $\mathrm{L}$. & Papilionaceae & UHI (3397) & 15 & Ominsinminsin \\
\hline 9 & Parquetina nigrescens & Periplocaceae & UHI (16026) & 15 & Ogbo \\
\hline 10 & Calotropis procera Ait. T. & Asclepiadaceae & UHI (9495) & 13 & Bomubomu \\
\hline 11 & Chenopodium ambrossiodes L. & Chenopodiaceae & UHI (709) & 12 & Arunpale \\
\hline 12 & Ocimum canum $\mathrm{L}$ & Lamiaceae & UHI (9492) & 11 & Efinrin wewe \\
\hline 13 & Bryophyllum pinnatum Salisb & Crassulaceae & $\mathrm{UHI}(400)$ & 11 & Abamoda \\
\hline 14 & Senna podocarpa L & Caesalpinaceae & $\mathrm{UHI}(3146 \mathrm{~A})$ & 11 & Asunran funfun \\
\hline 15 & Plumbago zeylanica $\mathrm{L}$ & Plumbaginaceae & UHI (6676) & 11 & Inabiri \\
\hline 16 & Cyatula prostrata Blume & Amaranthaceae & $\mathrm{UHI}(803)$ & 10 & Sawerepepe \\
\hline 17 & Peperromia pellucida (L.) Kunth & Piperaceae & UHI (245) & 9 & Rinrin \\
\hline 18 & Heliotropium indicum $\mathrm{L}$. & Boraginaceae & UHI (13279 & 9 & Ogbe akuko \\
\hline 19 & Cymbopogon citratus Stapf & Graminae/Poaceae & UHI (10232) & 9 & Kokooba \\
\hline 20 & $\begin{array}{l}\text { Solanum dasyphyllum Schumach \& } \\
\text { Thonn }\end{array}$ & Solanaceae & $\mathrm{UH}(1543)$ & 9 & Boboawodi \\
\hline 21 & Hibiscus surathensis L & Malvaceae & UHI (2360) & 9 & Sinkinrinmini \\
\hline 22 & Senna occidentalis L & Caesalpinaceae & UHI (16020) & 9 & Abo rere \\
\hline 23 & Gossypium arboretum L & Malvaceae & UHI (15293) & 9 & Owo akese \\
\hline 24 & Morinda lucida L & Rubiaceae & UHI (5672) & 9 & Oruwo \\
\hline 25 & Euphorbia laterifolia L & Euphorbiaceae & & 9 & Enuopiri \\
\hline 26 & Azadirachta indica A.Juss & Meliaceae & UHI (16012) & 8 & Dongoyaro \\
\hline 27 & Ageratum conyzoides L. & Asteraceae & UHI (15462) & 8 & Imi esu \\
\hline 28 & Boerhvia diffusa $\mathrm{L}$. & Nyctaginaceae & UHI (16004) & 8 & Etiponola \\
\hline 29 & Euphobia poisoni $\mathrm{L}$ & Euphorbiaceae & UHI (2647) & 8 & Enukokure \\
\hline 30 & Rhus longipee L & Anacardiaceae & & 8 & Orijin \\
\hline 31 & Pergularia deamia Choir & Asclepiadaceae & UHI (13812) & 8 & ljoyun \\
\hline 32 & Citrus aurantifolia Swingle & Rutaceae & UHI (4575) & 7 & Orombo wewe \\
\hline 33 & Usteria guineense Wild & Loganiaceae & & 7 & Esinsin ile \\
\hline 34 & Momordica charantia L & Curcubitaceae & UHI (15427) & 7 & Ejinrin wewe \\
\hline 35 & Aerva lanata Forsk. & Amaranthaceae & UHI (734) & 7 & Ewe owo \\
\hline 36 & Sansevieria liberica Thunb & Agavaceae & & 6 & Oja Ikooko \\
\hline 37 & Ricinus communis $\mathrm{L}$ & Euphorbiaceae & UHI (2804) & 6 & Lara pupa \\
\hline 38 & Lactuca taraxaxifolia L & Asteraceae & UHI (6392) & 6 & Yanrin \\
\hline 39 & Lawsonia inermis L & Lythraceae & UHI (971B) & 6 & Laali \\
\hline 40 & Bidens pilosa $\mathrm{L}$ & Asteraceae & UHI (14898) & 6 & Akisan \\
\hline 41 & Acanthus montanus L & Acanthaceae & UHI (14816) & 6 & Ahon ekun \\
\hline 42 & Cassytha filiformis L & Lauraceae & UHI (16016) & 6 & Omonigelegele \\
\hline 43 & Musa sapientium L & Musaceae & $\mathrm{UHI}(7953 \mathrm{~B})$ & 6 & Ogede omini \\
\hline 44 & Euphorbia kamerunica Pax & Euphorbiaceae & UHI (2634) & 5 & Oro agogo \\
\hline 45 & Starchytarpheta cayanensisVahl. & Verbanaceae & UHI (7755) & 5 & Iru-eku \\
\hline 46 & Jatropha multifeda $\mathrm{L}$ & Euphorbiaceae & UHI (2702) & 5 & Ogege \\
\hline 47 & Crinum jagus $\mathrm{L}$ & Amarilydaceae & UHI (8701) & 5 & Ogede odo \\
\hline 48 & Datura metel L & Solanaceae & UHI (6769) & 5 & Apakan \\
\hline 49 & Mimosa pudica $\mathrm{L}$ & Mimosaceae & UHI (16136) & 5 & Adegbile \\
\hline 50 & Mirabilis jalapa $\mathrm{L}$ & Nyctaginaceae & UHI (1188) & 5 & Tanoposo \\
\hline 51 & Newboldia laevis Seureau ex Bureau & Bignoniaceae & UHI (15486) & 5 & Akoko \\
\hline
\end{tabular}




\begin{tabular}{|c|c|c|c|c|c|}
\hline 52 & Spondias monbin L & Anacardiaceae & UHI (4837) & 5 & lyeye \\
\hline 53 & Nicotiana tabacum $\mathrm{L}$ & Solanaceae & UHI (14702) & 5 & Ewe taba \\
\hline 54 & Sphenocentrum jollyanum Pierre & Menispermaceae & UHI (9483) & 4 & Akerejupon \\
\hline 55 & Uraria picta (Jacq.) DC & Papilionaceae & UHI (15174) & 4 & Alupayida \\
\hline 56 & Carica papaya L & Caricaceae & UHI (14729) & 4 & Ibepe \\
\hline 57 & Holarrhena floribunda $R \cdot B r$. & Apocynaceae & UHI (14597) & 4 & Ako-ire \\
\hline 58 & Ficus thoningii Blume & Moraceae & UHI (4218) & 4 & Odan \\
\hline 59 & Ipomea asarifolia L & Convolvulaceae & UHI (16022) & 4 & Omu \\
\hline 60 & Zingiber officinale Roscoe & Zingiberaceae & & 4 & Atale funfun \\
\hline 61 & Lagenaria breviflora Ser. & Curcubitaceae & UHI (13226) & 4 & Tagiri \\
\hline 62 & Laggera aurita (Linn) DC & Asteraceae & UHI (6063) & 4 & Taba ogbugbu \\
\hline 63 & Bixa orelana L. & Bixaceae & UHI (1232) & 4 & Aje, \\
\hline 64 & Loncocarpus cyanensis Kunth. & Papilionaceae & UHI (3962) & 4 & Elu \\
\hline 65 & Harisona abysinica Oliv. & Simaroubaceae & UHI (4620) & 4 & Arujeran \\
\hline 66 & Chasmanthera dependens Hochst & Menispermaceae & UHI (13420) & 4 & Atoo \\
\hline 67 & Citrus aurantium Swingle & Rutaceae & & 3 & Jaganyin \\
\hline 68 & Curcuma longa Roxb & Zingiberaceae & & 3 & Atale pupa \\
\hline 69 & Dracaena mannii Vand ex $\mathrm{L}$ & Agavaceae & UHI (13945) & 3 & Peregun \\
\hline 70 & Solanum torvum Sw. & Solanaceae & UHI (16005) & 3 & Sokopeye \\
\hline 71 & Rauwolfia vomitoria Afzel & Apocynaceae & UHI (10286) & 3 & Asofeyeje \\
\hline 72 & Cochorus olitorious L & Tiliaceae & UHI (2119B) & 3 & Ewedu \\
\hline 73 & Costos afer $\mathrm{L}$ & Costaceae & UHI (15479) & 3 & Teteregun \\
\hline 74 & Anthocleista vogelii Planchon & Loganiaceae & UHI (14626) & 3 & Saposapo \\
\hline 75 & Eudenia trifoliolata Schum \&Thonn. & Capparidaceae & $\mathrm{UHI}(327)$ & 3 & Logbokiyan \\
\hline
\end{tabular}

Plants not observed in at least 3 TMP homesteads (representing $5 \%$ of total respondents) were not regarded as been cultivated by the TMPs.

Table 7: Plants frequently collected from the forest/wild sources by TMPs in Ayedaade LGA, Osun State, Nigeria.

\begin{tabular}{|l|l|l|l|l|l|}
\hline No & \multicolumn{1}{|c|}{ Name } & \multicolumn{1}{|c|}{ Family } & \multicolumn{1}{|c|}{$\begin{array}{c}\text { Voucher } \\
\text { number }\end{array}$} & $\begin{array}{c}\text { Collection } \\
\text { frequency } \\
\text { (\%) }\end{array}$ & \multicolumn{1}{c|}{ Yoruba name } \\
\hline 1 & Alstonia boonei R.Br. & Apocynaceae & UHI (13827) & 21 & Ahun \\
\hline 2 & Khaya senegalensis A.Juss & Meliaceae & UHI (4687) & 21 & Oganwo \\
\hline 3 & Rauwolfia vomitoria Afzel & Apocynaceae & UHI (5190) & 19 & Asofeyeje \\
\hline 4 & Morinda lucida Benth & Rubiaceae & UH(14650) & 19 & Oruwo \\
\hline $\mathbf{5}$ & Anthocleista vogelii Planchon & Loganiaceae & UHI (14626) & 13 & Saposapo \\
\hline $\mathbf{6}$ & Tetrapleura tetraptera Benth & Mimosaceae & UHI (3389A) & 11 & Aridan \\
\hline $\mathbf{7}$ & Abrus precatorius L. & Caesalpinaceae & UHI (2634) & 10 & Ominsiminsin \\
\hline 8 & Mormordica charantia L. & Curcubitaceae & UHI (15427) & 9 & Ejinrin wewe \\
\hline 9 & Milicia excelsia (Welw.) Benth & Moraceae & UHI (14754) & 9 & Iroko \\
\hline 10 & Lecaniodiscus cupanioides Planchon & Sapindaceae & UHI (14594) & 8 & Aaka \\
\hline 11 & Spondias mombin L. & Anacardiaceae & UHI (4837) & 8 & Iyeye \\
\hline 12 & Garcinia cola L & Gutiferae & UHI (13184) & 8 & Orogbo \\
\hline 13 & Afzellia africana Smith ex Per & Caesalpinaceae & UHI (2932) & 8 & Apa \\
\hline 14 & Kigelia africana (Lam.) Benth & Bignoniaceae & UHI (7304) & 7 & Pandoro \\
\hline 15 & Terminalia superba Engl. \& Diels & Combretaceae & UHI (13724) & 7 & Afara \\
\hline 16 & Olax subscopoidea L & Olacaceae & UHI (4402A) & 7 & Ifon \\
\hline 17 & Harungana madagascariensis Lam. & Harungaceae & UHI (16056) & 7 & Amuje \\
\hline 18 & Mallotus oppositifolius Muell.Arg & Euphorbiaceae & UHI (14680) & 7 & Orokoro \\
\hline 19 & Vernonia amygdalina Schreber & Asteraceae & UHI (6532) & 6 & Ewuro \\
\hline 20 & Secamone afzelii R.Br. & Asclepiadaceae & UHI (14930) & 6 & Ailu \\
\hline 21 & Bridelia ferruginea Wild & Euphorbiaceae & UHI (14619) & 6 & Ira odan \\
\hline
\end{tabular}




\begin{tabular}{|c|c|c|c|c|c|}
\hline 22 & Ageratum conyzoides L & Asteraceae & UHI (15462) & 6 & Apasa, Imi-esu \\
\hline 23 & Senna alata $\mathrm{L}$ & Caesalpinaceae & UHI (13918) & 5 & Asunran pupa \\
\hline 24 & Cissampelos owariensis L & Menispermaceae & UHI (14920) & 5 & Jenjoko \\
\hline 25 & Abrus canascens $\mathrm{L}$ & Papilionaceae & & 5 & $\begin{array}{l}\text { Ominsinminsin } \\
\text { gogoro }\end{array}$ \\
\hline 26 & Piper guinneense Schum \& Thonn & Piperaceae & UHI (13153) & 5 & lyere \\
\hline 27 & Azadirachta indica A.Juss & Meliaceae & UHI (16012) & 5 & Dongoyaro \\
\hline 28 & Aerva lanata Forssk. & Amaranthaceae & UHI (734) & 5 & Ewe owo \\
\hline 29 & Carica papaya L & Caricaceae & UHI (14729) & 5 & Ibepe \\
\hline 30 & Cissus populnea Guill \& Perr & Vitaceae & UHI (14883) & 4 & Ogbolo \\
\hline 31 & $\begin{array}{l}\text { Zanthoxylum xanthoxyloides Lam } \\
\text { Waterman }\end{array}$ & Rutaceae & $\mathrm{UHI}(4605)$ & 4 & Orin ata \\
\hline 32 & Parkia biglobosa (Jacq.) G.Don & Mimisaceae & UHI (3354A) & 4 & Igbaru \\
\hline 33 & Aframonum melegueta Schuman & Zingiberaceae & UHI (13309) & 4 & Atare \\
\hline 34 & Plumbago zeylanica $L$. & Plumbaginaceae & UHI (6676) & 4 & Inabiri \\
\hline 35 & Mondia whitei Skeels & Periplocaeae & UHI (5253) & 4 & Isirigun \\
\hline 36 & Ceiba pentandra L & Bombacaceae & UHI (2260) & 4 & Araba \\
\hline 37 & Ocimum gratissimum $\mathrm{L}$ & Lamiaceae & UHI (14849) & 4 & Efinrin foromoba \\
\hline 38 & $\begin{array}{l}\text { Piptadeniastrum } \\
\text { (Hook.f.)Brenam }\end{array}$ & Mimosaceae & UHI (3360) & 4 & Agbonyin \\
\hline 39 & Xylopia aethiopica A.Rich & Annonaceae & UHI (13153) & 4 & Eeru \\
\hline 40 & Mangifera indica $\mathrm{L}$ & Anacardiaceae & UHI (4820) & 4 & Mangoro \\
\hline 41 & Bidens pilosa $\mathrm{L}$ & Asteraceae & UHI (14898) & 4 & Akisan \\
\hline 42 & Uvaria picta (Jacq.)DC & Papilionaceae & UHI (15174) & 4 & Alupayida \\
\hline 43 & Celtis zenkeri $\mathrm{L}$ & Ulmaceae & UHI (1872) & 3 & Ita \\
\hline 44 & Cordia millenii Baker & Boraginaceae & UHI (6731) & 3 & Omo \\
\hline 45 & Euphobia kamerunica Pax & Euphorbiaceae & UHI (2634) & 3 & Oro agogo \\
\hline 46 & Elaeis guinensis Jacq. & Palmae & UHI (8964) & 3 & Ope \\
\hline 47 & Monodora tennuifolia Dunal & Annonaceae & UHI (059) & 3 & Aworoso \\
\hline 48 & Treculia africana Decne & Moraceae & $\mathrm{UHI}(4255 \mathrm{~A})$ & 3 & Afon \\
\hline 49 & Citrullus colocynthis Schrader & Curcubitaceae & UHI (1338) & 3 & Egusi bara \\
\hline 50 & Acacia farnesiana Wild & Mimosaceae & UHI (3203) & 3 & Booni \\
\hline 51 & Ficus thonningii Blume & Moraceae & UHI (4166) & 3 & Opoto \\
\hline 52 & Ocimum canum $\mathrm{L}$ & Lamiaceae & UHI (9492) & 3 & Efinrin wewe \\
\hline 53 & Erythrophleum suaveolens Brenan & Caesalpinaceae & UHI (15096) & 3 & Obo \\
\hline 54 & Pergularia daemia $\mathrm{L}$ & Asclepiadaceae & UHI (13812) & 3 & ljoyun \\
\hline 55 & Mirabilis jalapa $\mathrm{L}$ & Nyctaginaceae & UHI (1188) & 3 & Tanoposo \\
\hline 56 & Senecio abysinicus L & Asteraceae & UHI (6460) & 3 & Amunimuye \\
\hline 57 & Crinum jagus L & Amarilydaceae & UHI (8701) & 3 & Ogede odo \\
\hline 58 & Newboldia laevis Seeman ex Bureau & Bignoniaceae & $\mathrm{UHI}(15486)$ & 3 & Akoko \\
\hline 59 & Costos afer $\mathrm{L}$ & Costaceae & & 3 & Teteregun \\
\hline 60 & Euphobia poisonii L & Euphorbiaceae & UHI (2647) & 3 & Enukokure \\
\hline 61 & Syncephalum dulcificum DC & Asteraceae & & 3 & Agbayun \\
\hline 62 & Irvingia gabonensis Bailon & Irvingaceae & UHI (13113) & 3 & Oro \\
\hline 63 & Eudenia trifoliolata Schum. \& Thonn & Capparidaceae & $\mathrm{UHI}(327)$ & 3 & Logbokiyan \\
\hline 64 & Anona senegalensis $\mathrm{L}$ & Annonaceae & $\mathrm{UHI}(13021)$ & 3 & Abo \\
\hline
\end{tabular}

Plants not mentioned by at least 3 TMPs (representing 5.5\% of the total respondents) were not included in the plants usually collected from wild sources. 
Table 8: Plants usually purchased by TMPs in Ayedaade LGA, Osun State, Nigeria.

\begin{tabular}{|c|c|c|c|c|c|}
\hline No & Name & Family & $\begin{array}{l}\text { Voucher } \\
\text { number }\end{array}$ & $\begin{array}{l}\text { Frequency } \\
\text { of purchase }\end{array}$ & $\begin{array}{l}\text { Local name - } \\
\text { Yoruba }\end{array}$ \\
\hline 1 & Xylopia aethiopica (Dun.) A.Rich & Annonaceae & UHI (14625) & 29 & Eeru \\
\hline 2 & Aframomum melegueta Schuman & Zingiberaceae & UHI (13309) & 27 & Atare \\
\hline 3 & Piper guineense Schum \& Thonn & Piperaceae & UHI (13153) & 24 & Iyere \\
\hline 4 & Tetrapleura tetraptera Benth & Mimosaceae & UHI (3389A) & 22 & Aridan \\
\hline 5 & Erythrophleum suaveolens Brenan & Caesalpinaceae & UHI (15096) & 19 & Obo \\
\hline 6 & Allium ascalonicum Auc.Non.L & Alliaceae & & 12 & $\begin{array}{l}\text { Alubosa } \\
\text { elewe }\end{array}$ \\
\hline 7 & Zingiber officinale Roscoe & Zingiberaceae & & 10 & Atale funfun \\
\hline 8 & Cremaspora triflora Thonn. K.Schum & Rubiaceae & UHI (14651) & 8 & Ina \\
\hline 9 & Acacia farnesiana Wild & Mimosaceae & $\mathrm{UHI}(3203)$ & 8 & Booni \\
\hline 10 & Securidaca longipedunculata Fres. & Polygalaceae & UHI (13029) & 8 & Ipeta \\
\hline 11 & Khaya senegalensis A.Juss & Meliaceae & UHI (14716) & 7 & Oganwo \\
\hline 12 & Allium sativum $\mathrm{L}$ & Alliaceae & & 7 & Ayu \\
\hline 13 & Capsicum fructescens $\mathrm{L}$ & Solanaceae & UHI (14881) & 7 & Ata ijosin \\
\hline 14 & Syzigium aromaticum $\mathrm{L}$ & Myrtaceae & & 6 & Kannafuru \\
\hline 15 & Terminalia avicenoides Guill \& Per & Combretaceae & UHI (15428) & 6 & Idi \\
\hline 16 & Mondia whitei Skeels & Periplocaceae & $\mathrm{UHI}(5253)$ & 5 & Isirigun \\
\hline 17 & Anogeisus leocarpus Guillemin \& Perrottet & & & 5 & Ayin \\
\hline 18 & Garcinia cola L & Gutiferae & UHI (13184) & 5 & Orogbo \\
\hline 19 & Aristolochia ringens $\mathrm{L}$ & Aristolochiaceae & UHI (211) & 4 & Akogun \\
\hline 20 & Bridelia ferruginea Wild & Euphorbiaceae & UHI (14619) & 4 & Ira odan \\
\hline 21 & Sphenocentrum jollyanum Pierre & Menispermaceae & $\mathrm{UHI}(14900)$ & 4 & Akerejupon \\
\hline 22 & Piliostigma thonningii Hochst & Caesalpinaceae & UHI (14885) & 4 & Abafe \\
\hline 23 & Anona senegalensis $\mathrm{L}$ & Annonaceae & UHI (13021) & 4 & Abo \\
\hline 24 & Uvaria picta (Jacq.) DC & Papilionaceae & UHI (15174) & 4 & Alupayida \\
\hline 25 & Zanthoxylum xanthoxyloide L & Rutaceae & UHI (4605) & 4 & Orin ata \\
\hline 26 & Picralima nitida Pierre & Apocynaceae & UHI (5165) & 4 & Abeere \\
\hline 27 & Senecio abysinicus $\mathrm{L}$ & Asteraceae & $\mathrm{UHI}(6460)$ & 4 & Amunimuye \\
\hline 28 & Abrus precatorius $\mathrm{L}$ & Papilionaceae & $\mathrm{UHI}(3397)$ & 4 & Oju ologbo \\
\hline 29 & Sorghum bicolor Moench & Poaceae & UHI (10976) & 3 & Poporo oka \\
\hline 30 & Euphorbia kamerunica Pax & Euphorbiaceae & UHI (2634) & 3 & Oro agogo \\
\hline 31 & Curcuma longa Valeton & Zingiberaceae & & 3 & Atale pupa \\
\hline 32 & Butyrospermum paradoxii Gaertner f. & Sapotaceae & UHI (15426) & 3 & Emi \\
\hline 33 & Cyathula prostrate Blume & Amaranthaceae & & 3 & Sawerepepe \\
\hline 34 & Rauwolvia vomitoria Afzel & Apocynaceae & UHI (5190) & 3 & Asofeyeje \\
\hline
\end{tabular}

Plants not mentioned by at least 3 TMPs (representing $5.5 \%$ of the total respondents) were not included in the plants usually purchased from herbsellers.

\section{DISCUSSION}

The challenges of rural urban migration in search of social infrastructures obviously posed a threat on the future of traditional medicine practice in the study area; the interests of younger generations in acquisition of indigenous knowledge and skill in the use of plant resources for health care delivery are waning. Most of the TMPs are above 40 years as also observed in Ethiopia by Bekalo et al, 2009. It is unlikely that this group of elders would migrate into another occupation, unlike the younger ones who might change occupation with time.

The study revealed that about 35 TMPs spend at least USD210 dollars daily to procure herbs and this will amount to about USD 2,520/year in an area where there are about 35 TMPs only (Fig.2). This is an indication that medicinal plants cultivation as a tool of conservation has a good market potential in improving socio-economic factors of peasant farmers in the study area. Cultivation of medicnal plants will undoubtedly generates financial empowerment and employment opportunities, (Lange, 1988 and Sher et al., 2010). In the 36 states of Nigeria, this implies a 
huge estimate of $(90,720$ USD) potential income from medicinal plants if cultivated. This high demand of medicinal plants could lead to shortage of plant resources for traditional health care delivery if sustainable means of medicinal plant production are not put in place. Substantial extraction of medicinal plants has resulted in the depletion of existing populations of many valuable species in Ayedaade Local Government area, Osun state, Nigeria (Table 4). The study indicates a significant positive correlation of $(0.355, \rho \leq 0.01)$ between scarcity of medicinal plants and average daily purchase of herbs by TMPs. More money is expended on herbal material with increasing scarcity. Increase in the distance travel by TMPs to obtain their plant materials from the wild has resulted in the corresponding increase in the average daily expenses on herbs. This is expressed in the significant positive correlation of $(0,382, \rho \leq 0.01)$. Different studies had observed loss of forest land to agriculture, urbanization and overexploitation of forest resources as factors forcing traditioner healers to travel long distances before obtaining plant materials for their preparation (Omobuwajo et al., 2008, Kisangau et al., 2004, Tomlison, 1998, Quedraogo, 1995). Scarcity is further aggravated by local plant collectors who do not employ sustainable harvesting technique for possible regrowth of the harvested plant parts in most cases, wounds and injuries are inflicted, which in most cases prevents recovery and eventual death of the plants, especially when stem barks and roots are harvested from wild sources. The activities of medicinal plants suppliers exert more pressure on these plant resources in the study area. .

It was observed that no noticeable deliberate efforts have been put into medicinal plants cultivation and conservation in the study area (Table 4), as observed by Oladele et al., 2008. However, about $86.8 \%$ of the TMP's expresed their interest to patronize medicinal plants farm if available. This willingness will assist in the conservation of these important natural resources by reducing undue pressure on the wild sources. Proper information dissemination will be required for such farms to avoid marketing problem. Rao et al., (2004), observed that lack of land is the most significant constraint to medicinal plant cultivation, this is represented by $(47.3 \%)$ of TMPs in the study. Small Land holding and land tenure system makes sourcing for land difficult especially in developing countries. Lack of time to maintain medicinal plants farm is another major constraint given by the informants, about $21.8 \%$ of the TMPs in the study population had no time to maintain such farms. This may be acceptable because they have to be available in their premises always.

CONCLUSION: Traditional medicine has a high impact on rural as well as urban communities due to demand for their products. This places pressure on the traditional medicine practitioners and ultimately on the medicinal plants in their habitats. The TMPs interviewed have considerable awareness of plant conservation needs due to their scarcity hence, they developed the strategy of growing some frequently used plants ex situ in their premises (Table 6) to sustain traditional health care delivery for decades . The plants listed in Table $7 \& 8$ are candidate plants that require conservation needs in the study area, both in situ and ex situ techniques should be encouraged for fast growing as well as slow growing species. Sustainable harvesting techniques for plant parts need to be encouraged among TMPs and farmers for the scarce species. Conservation of plant diversity requires the effort of all stake holders including the TMPs who must be at the focal point of any initiative for conservation as a form of joint management of natural resources. Establishment of community medicinal plants farm as a form of participatory approach to sustainable exploitation of medicinal plants will possibly curb overexploitation which threatens forests and the people dependent upon them (Cunningham, 1997). Incorporation of medicinal plants in agricultural fields will help in no small measure to increase supply and conservation of important medicinal plants in the study area.

\section{REFERENCES}

Bekalo, T.H., S.D Woodmatas and Z.A Woldemariam, 2009. An ethnobotanical study of medicinal plants used by local people in the lowlands of Konta Special Woreda, Southern nations, nationalities and peoples regional state, Ethiopia. Journal of Ethnobiology and Ethnomedicine 5:26 DOI:10.1186/1746-4269-5-26, Available from: http://www.ethnobiomed.com/content/5/1/26 ,[Accessed 30th, July 2010]

Bhat, R.B., E.O Etejere and V.T Oladipo.1990. Ethnobotanical studies from Central Nigeria. Economic Botany 44 (3): 382-390

Bodeker, G. 1994. Traditional Health Knowledge and Public Policy. Natural Resources 30 (2): 91-106

Cunningham, A.B. 1993. African Medicinal Plants: Setting Priorities at the interface between Conservation and 
Primary Healthcare. People and Plants Working Paper 1 , Paris.

Cunningham, A.B. 1997. An Africa-wide overview of medicinal plants harvesting, conservation and health care. Non-Wood Forest Products No. 11: Medicinal plants for forest conservation and health care, FAO, Rome, Italy.

De Silva, 1997. Industrial Utilization of medicinal plants in developing countries. (In) Bodeker G., K.K.S Bhat, J.Burkey and P.Vantomme. (Eds.), Medicinal plants for forest conservation and healthcare, Non-Wood Forest Product No. 11: FAO Rome Italy.

Federal Republic of Nigeria. 2007. 2006 Census, Official Gazette No. 24 Vol. 94. Publisher: Federal Govt Printer, Lagos, Nigeria FGP 71/ 52007/2,500(DL24)

Gupta, R. 1998. Integration of medicinal plants cultivation in forest and forest plantations in northwest Himalaya, pp 59-67 In Agro forestry systems: A new challenge in Indian Society of tree scientists, Solan, India

Hassan Sher, F. Hussain and H.Sher. 2010. Ex-situ Management Study of some high value medicinal plant species in Swat, Pakistan. Ethnobotanical Research and Applications 8: $017 \quad-\quad 024$ www.ethnobotanyjournal.org/vol8/i1547-3465-08017.pdf ,[Accessed 25th Sept, 2010]

Kisangau, D. and J. O Kokwaro. 2004. Use of Medicinal Plants: Kenya in Sharing Innovative Experiences, United Nations Development Programme, New York, 9: 60

Lange, D.1988. Europe's Medicinal and Aromatic plants: Their use, trade and conservation. TRAFFIC, Cambridge. UK

Mahonge, C.P.I., J .V Nsenga, Mtangi and A.C Matte. 2006. Utilization of medicinal plants by Walguru people in east Uluguru mountains, Tanzania. African Journal of Traditional \& Alternative and Alternative Medicine (4): 121-134

Oladele, A.T., S.A. Adesanya and O.R Omobuwajo. 2008. Effects of two inorganic fertilizers on volatile oil and biomass yields of Cymbopogon citratus (DC). Stapf. in Ile-Ife, Nigeria. Nigeria Journal of Natural Products and Medicine vol.12. 2008: 9-12.

Omobuwajo, O. R., G .O. Alade and A. Sowemimo. 2008. Indigenous Knowledge and practices of women herb sellers of Southwestern Nigeria. Indian Journal of Traditional Knowledge 7(3) : 505-510

Pramono, E. 2002. The Commercial use of Traditional knowledge and Medicinal Plants in Indonesia. Multistakeholder Dialogue on Trade, Intellectual Property and Biological Resources in Asia, BRAC Rajendrapur, Bangladesh, 1-13

Quedraogo, A. 1995. In situ Conservation and Sustainable use of Forest Species, (In) In-situ Conservation and Sustainable use of plant Genetic Resources for Food and Agriculture in Developing Countries, Engels JMM (IPDRI, Rome, Italy and DSE Feldafeng, Germany), 101-103

Rao, M.R., M.C. Palada and B. N. Becker. 2004. Medicinal and aromatic plants in Agro forestry systems (61) : 107-122

Sofowora, A. 1993. Medicinal Plants and Traditional Medicine in Africa, (Spectrum Books Limited, Ibadan, Nigeria), 249-258

Schippman, U., D .J. Leaman and A. B. Cunningham. 2002. The impacts of cultivation and gathering of medicinal plants of biodiversity. Global trends and issues, (In) Biodiversity and Ecosystem. Approaches in Agriculture, forestry, and fisheries. 9th regular sessions of the commission on Genetic Resources for food and Agriculture. FAO Rome, Italy pp 1-21

SPSS 16.0 for Windows, 2007. Microsoft Corporation, USA

Tomlison, R. 1998. Promoting the World Wide Use of Medicinal Plants, in Medicinal Plants and their role in Health and Biodiversity, edited by Tomlison $\mathrm{R}$ and Akerele $\mathrm{O}$ (University of Pennsylvania, Philadephia, USA), $9-12$ 